\title{
Correlating structure and morphology to device performance of molecular organic donor-acceptor photovoltaic cells based on Diindenoperylene (DIP) and $\mathbf{C}_{60}$
}

M. Gruber, M. Rawolle, J. Wagner, D. Magerl, U. Hörmann, J. Perlich, S. V. Roth, A. Opitz, F. Schreiber, P. Müller-Buschbaum, and W. Brütting*

[*] Prof. W. Brütting, M. Gruber, J. Wagner, U. Hörmann

Institut für Physik

Universität Augsburg

Universitätsstr.1, 86135 Augsburg, Germany

E-mail: wolfgang.bruetting@physik.uni-augsburg.de

M. Rawolle, D. Magerl, Prof. P. Müller-Buschbaum

Physik-Department, Lehrstuhl für funktionelle Materialien

Technische Universität München

James-Frank-Str.1, 85748 Garching, Germany

Dr. J. Perlich, Dr. S. V. Roth

HASYLAB at DESY

Notkestr.85, 22603 Hamburg, Germany

\section{A. Opitz}

Institut für Physik, Supramolekulare Systeme

Humboldt-Universität zu Berlin

Brook-Taylor-Straße 6, 12489 Berlin, Germany

Prof. F. Schreiber

Institut für Angewandte Physik

Universität Tübingen

Auf der Morgenstelle 10, 72076 Tübingen, Germany

Keywords: diindenoperylene, organic solar cells, GISAXS, thin film morphology

\begin{abstract}
The performance of organic photovoltaic cells (OPVCs) shows a critical dependence on morphology and structure of the active layers. In small molecule donor/acceptor (D/A) cells fabrication parameters, like substrate temperature and evaporation rate, play a significant role for crystallization and roughening of the film. In particular, the fraction of mixed material at the interface between donor and acceptor is highly relevant for device performance. While an ideal planar heterojunction (PHJ) exhibits the smallest possible interface area resulting in suppressed recombination losses, mixed layers suffer strongly from recombination but show higher exciton dissociation efficiencies.
\end{abstract}


In this study we investigate PHJ and planar-mixed heterojunction (PM-HJ) solar cells based on diindenoperylene (DIP) as donor and $\mathrm{C}_{60}$ as acceptor, fabricated under different growth conditions. Grazing incidence small angle X-ray scattering (GISAXS), X-ray reflectometry (XRR) and atomic force microscopy (AFM) are used to obtain detailed information about inand out-of-plane structures and topography. In that way we find that surface and bulk domain distances are correlated in size for PHJs, while PM-HJs show no correlation at all. The resulting solar cell characteristics are strongly affected by the morphology, as reorganizations in structure correlate with changes in the solar cell performance.

\section{Introduction}

Charge carrier and exciton transport are important issues in the field of organic semiconductor devices. Owing to weak intermolecular coupling and low dielectric constants the binding energies of photo-generated Frenkel-excitons (typically in the range of a few tenths of an eV for materials relevant in photovoltaics) are too high to be separated by thermal energy at room temperature. ${ }^{[1]}$ Because light-induced excitons are usually localized on one single molecule, transport is dominated by exciton-hopping resulting in short exciton diffusion lengths (EDLs) of only a few nanometers, depending on the structural order of the material. Many commonly used materials are dominated by amorphous growth showing EDLs of typically $10 \mathrm{~nm}$ or less, ${ }^{[2-4]}$ while crystalline materials like diindenoperylene (DIP) can reach EDLs of up to 100 $\mathrm{nm}$ in thermally evaporated thin films. ${ }^{[5]}$

Not only is exciton transport hindered in organic semiconductors, but also is the charge carrier mobility. Depending on the details of film growth, differences in mobility can be huge. While amorphous materials show mobilities of typically $10^{-3} \mathrm{~cm}^{2} / \mathrm{Vs}$ or less at room temperature due to hopping transport of charge carriers in a Gaussian density of states, highly crystalline films almost show band-like transport with mobilities that are several orders of magnitude higher. $^{[6,7]}$

For efficient exciton dissociation, Tang et al. introduced the donor/acceptor (D/A) concept. ${ }^{[8]}$ Comprising such a heterojunction several architectures for organic photovoltaic cells (OPVCs) have been proposed with varying amount of mixed material at the interface leading to increased exciton dissociation probability, but also the tendency for enhanced charge carrier recombination for larger interfacial areas. ${ }^{[9-11]}$ One extreme can be found in the ideal planar heterojunction (PHJ) without any fraction of mixed material and a flat interface. To 
overcome the bottleneck of short EDLs, a D:A mixture forming a bulk heterojunction (BHJ) exhibits the largest possible interface leading to a reduced pathway for excitons to reach the dissociating interface. ${ }^{[12-14]}$ In the case of BHJs two types have to be distinguished. While the combination of two structurally compatible molecules allows for the formation of one molecularly mixed phase and in some cases even an ordered mixed crystal is possible, BHJs with structurally unlike molecules in shape normally form phase-separated structures. ${ }^{[15,16]}$ Both concepts of BHJs have the advantage of providing short distances for excitons to reach the interface within their EDL. However, the absence of percolation paths in molecularly mixed crystals causes severe problems for charge carrier transport towards the electrodes. ${ }^{[15,16]}$ Thus, reasonable results, when applied in solar cells, can only be achieved with PHJs, phase separated BHJs and a combination of both concepts in so-called planarmixed heterojunctions (PM-HJs).

In our study we investigate the impact of morphology on OPVCs with highly crystalline materials, namely diindenoperylene (DIP) as donor and $\mathrm{C}_{60}$ as acceptor. The effect of different growth conditions were studied in PHJs, which strongly affect the lateral grain size. In previous work on this material system it was shown that fabrication parameters like substrate temperature and evaporation rate play a significant role for crystallization and roughening of the film. ${ }^{[16-20]}$ However, also the fraction of mixed material at the interface between donor and acceptor is highly relevant. ${ }^{[21]}$ In the present study, we systematically investigate how changes of morphology, both in the bulk and at the surface of the film, affect crystallinity and solar cell performance.

The morphology in the volume of the films is investigated by grazing incidence small angle X-ray scattering (GISAXS), ${ }^{[22]}$ which provides information about domain distances in the bulk of the material. The volume information is compared with the domain distances of topography scans performed by atomic force microscopy (AFM). X-ray reflectometry (XRR) measurements are used to gain information on the crystallization behavior of both active materials in vertical direction. It is found that lateral domain distances in the bulk and at the surface of PM-HJs differ strongly. This is attributed to the pronounced phase separation of DIP and $\mathrm{C}_{60}$. Solar cells fabricated under comparable growth conditions and film thicknesses show increased $j_{\mathrm{SC}}$ in PM-HJs and increased FF in PHJs, respectively. These two effects compensate each other and result in nearly the same power conversion efficiency $\eta$ for both systems. 


\section{Experimental details}

\section{Sample preparation}

For structural and topographical measurements we used silicon substrates overgrown with a $320 \mathrm{~nm}$ thick thermal $\mathrm{SiO}_{2}$ layer, while solar cells were fabricated on indium tin oxide (ITO) coated glass substrates. As hole extraction layer for solar cells a $30 \mathrm{~nm}$ thick poly(3,4ethylenedioxythiophene) : poly(styrenesulfonate) (PEDOT:PSS, purchased from Clevios as Baytron P AI4083) film, was spin-coated on top of the ITO layer.

The donor DIP was purchased from S. Hirschmann (Univ. Stuttgart, Germany), the acceptor $\mathrm{C}_{60}$ from Creaphys in Dresden. The two photoactive molecular materials were twice purified by gradient sublimation before usage. Bathocuproine (BCP) was used as received from Sigma Aldrich for exciton blocking and protection against metal interdiffusion. ${ }^{[23,24]}$

The samples were prepared using organic molecular beam deposition at a base pressure smaller than $10^{-7}$ mbar. DIP and $\mathrm{C}_{60}$ were thermally evaporated in one chamber without breaking the vacuum at deposition rates of about $0.5 \AA / s$ for single material deposition. In the case of coevaporation of both materials the combined deposition rate was about $0.6 \AA / \mathrm{s}$. Further morphological and structural measurements were performed in air. For solar cell preparation the samples with $\mathrm{DIP} / \mathrm{C}_{60}$ were transferred to a glovebox that provides inert atmosphere without exposing the samples to air. In a second vacuum chamber directly connected to the glovebox the BCP layer and the aluminum electrode were evaporated with deposition rates of $0.3 \AA / s$ and $1.0 \AA / \mathrm{s}$, respectively.

Structural and topographical investigations were performed for neat DIP films deposited on differently heated silicon oxide substrates (room temperature (RT), $60{ }^{\circ} \mathrm{C}$ and $100{ }^{\circ} \mathrm{C}$ ) and on heterostructures, where DIP films grown on heated substrates $\left(100{ }^{\circ} \mathrm{C}\right)$ were subsequently covered with $\mathrm{C}_{60}$ with and without further substrate heating during evaporation of $\mathrm{C}_{60}$ (RT and $100{ }^{\circ} \mathrm{C}$ ). Layer thicknesses were $50 \mathrm{~nm}$ for DIP and $40 \mathrm{~nm}$ for $\mathrm{C}_{60}$. The corresponding OPVCs were fabricated using identical active layer stacks on top of pre-heated PEDOT:PSS covered ITO substrates.

Moreover, we fabricated a sample with PM-HJ architecture, i.e. a neat $4 \mathrm{~nm}$ thick evaporated DIP layer was followed by a $36 \mathrm{~nm}$ thick co-evaporated mixture of DIP and $\mathrm{C}_{60}$ in a 1:1 volume ratio, all deposited at a substrate temperature of $100{ }^{\circ} \mathrm{C}$. The corresponding solar cell included an additional layer of $6 \mathrm{~nm} \mathrm{C}_{60}$, which was evaporated on top of the blend to complete the PM-HJ architecture. For better insight into the mixed material this layer was left out for morphological and structural investigations. 
Exciton blocking and protection from metal incorporation in solar cells was granted by a $5 \mathrm{~nm}$ thick BCP layer covering the active layers topped by the electrical contact Al. In total, there were five different OPVCs.

\section{Film and solar cell characterization}

Grazing incidence small angle X-ray scattering (GISAXS) allows for obtaining statistically relevant structural information in the volume of thin films, with tunable sensitivity to the film surface by changing the incident angle. ${ }^{[22,25]}$ The basic setup is shown in Figure 1a: the X-ray beam impinges on the sample under a shallow incident angle $\alpha_{\mathrm{i}}$, typically well below $1^{\circ}$. The reflected scattering signal is detected on a two-dimensional detector, where the direct beam and the specularly reflected beam are each blocked by a beam stop. To obtain more information about lateral structures as probed parallel to the sample surface, a rod-like beam stop that blocks the intensity in z-direction around $q_{\mathrm{y}}=0$ can shield the detector in addition. GISAXS measurements have been performed at the beamline BW4 of HASYLAB at DESY, Hamburg, with a sample-to-detector distance of $2.2 \mathrm{~m}$ and a wavelength of $0.138 \mathrm{~nm} .{ }^{[26]}$ The incident angle was chosen as $0.35^{\circ}$, which is well above the critical angle for total reflection of the measured materials $\left(0.162^{\circ}\right.$ for DIP and $0.167^{\circ}$ for $\left.\mathrm{C}_{60}\right)$, enabling to probe structural information in the whole volume of the thin films with good resolution. The refractive index for DIP at the used X-ray wavelength is $n=1-4.02 \cdot 10^{-6}+\mathrm{i} 5.55 \cdot 10^{-9}$ and for $\mathrm{C}_{60}$ it is $\mathrm{n}=1$ $-4.25 \cdot 10^{-6}+\mathrm{i} 6.36 \cdot 10^{-9}$. The size of the moderately focused synchrotron beam was set to $(23 \times 36) \mu \mathrm{m}^{2}$ (vertical $\times$ horizontal), resulting in a footprint of the X-ray beam of $3.8 \mathrm{~mm}$ by $36 \mu \mathrm{m}$ on the sample. The scattered X-ray signal was detected with a MarCCD detector of $2048 \times 2048$ pixels with a pixel size of $(79.1 \mu \mathrm{m})^{2}$.

The data were analyzed by fitting lateral structure sizes with the effective interface approximation (EIA). ${ }^{[22]}$ To prove the validity of this approach, selected data sets were also simulated with the IsGISAXS software. ${ }^{[27]}$ The characteristic lateral structures obtained from simulation agree well with the ones of the effective interface approximation, but yield additional information about shape and distribution of the structures in the sample.

Surface topography was investigated using AFM (Thermo Microscopes Autoprobe CPResearch) in non-contact mode with an NSG30 (NT-MDT) tip with a pyramidal shape and $22-100 \mathrm{~N} / \mathrm{m}$ spring constant probing an area of $4 \mathrm{x} 4 \mu \mathrm{m}^{2}$. Fast Fourier transformation (FFT) of the signal provides distance distributions (see Fig. 1b). To interpret the results, data were averaged over the whole angle range of $\Theta$ (see Fig. 1) and fitted by Gaussian distributions. These fits provide the average domain distances of the associated AFM signal. 
XRR was performed for the samples on $\mathrm{SiO}_{2}$ using an XRD 3003 PTS (Seifert) with a wavelength of $0.154 \mathrm{~nm}$, a slit collimation system and a scintillation counter SZ 20/SE (Seifert). From these data, information about vertical domain sizes was obtained using the Scherrer equation.

Current-voltage characteristics were recorded from $-0.5 \mathrm{~V}$ to $1.5 \mathrm{~V}$ in $0.05 \mathrm{~V}$ steps using a source measure unit (Keithley 236 SMU) in dark and under illumination with a solar simulator (Oriel 300W with AM 1.5G filters) in a glovebox system with nitrogen atmosphere. The illumination intensity was approved by a calibrated silicon reference cell (RERA systems, PV Measurement Facility, Radboud University Nijmegen, area $1 \times 1 \mathrm{~cm}^{2}$ ).

\section{Results and Discussion}

\section{a) Influence of substrate temperature on DIP structure}

The AFM measurements of DIP films evaporated onto silicon substrates at different temperatures (see Figure 2a-c) show an increasing lateral DIP surface domain size and domain distance with increasing temperature. The morphology can be described as sphere- or granule-like objects. The lateral domain distances of these surface structures are extracted from FFT of the AFM images (see Figure 3). For all three temperatures two characteristic lateral distances are found. In addition, XRR (not shown) reveals a constant out-of-plane lattice parameter and a vertical coherence length essentially equivalent to the layer thickness, as derived from the width of the Bragg peak, independent of preparation temperature in the range studied. A similar behavior was reported in Ref. ${ }^{[20,28]}$.

The 2-dimensional GISAXS data (see Figure 4) exhibit a characteristic shape with Bragg rodlike intensity streaks originating from the lateral DIP structure. The position of the Bragg-rods moves towards smaller $q_{\mathrm{y}}$-values with increasing evaporation temperatures. To quantify this information, the horizontal line cuts (in $q_{\mathrm{y}}$-direction) at the critical angle of DIP are shown in Figure 5 with the corresponding fits based on the EIA. As in the FFT of the AFM data two characteristic lateral lengths are extracted. With increasing temperature the size of these characteristic lateral structures increases (see Table 1). Within the experimental error bars, the values of the small and large structures determined by GISAXS agree with the values as obtained from AFM measurements (also included in Tab. 1). Both show an increasing DIP domain distance with higher evaporation temperature. However, the GISAXS values tend to 
be slightly larger. Probably this is an effect of statistics, as AFM is a local probe at the surface, whereas GISAXS averages structural features in a larger sample volume. To gain information on structures perpendicular to the substrate, vertical cuts (in $q_{\mathrm{z}}$-direction) at different positions are performed. For vertical cuts at $q_{\mathrm{y}}=0$, representing very large structures in the resolution limit of the GISAXS experiment, no intensity oscillations are present for $q_{\mathrm{z}}$ values above the specular peak (approximately at $0.6 \mathrm{~nm}^{-1}$ ). Therefore these large, unresolved structures show no correlated roughness (i.e. the topography of the film differs from the topography of the underlying substrate) irrespective of the substrate temperatures.[29,30] In contrast, for small distances, i.e. at $q_{\mathrm{y}}$ values well outside the resolution limit, correlated roughness is observed for all substrate temperatures during evaporation. Correspondingly, in the $q_{\mathrm{z}}$-cut at the position of the intensity maximum in $q_{\mathrm{y}}$ intensity oscillations are present.

\section{b) DIP films on heated substrates, covered with $\mathrm{C}_{60}$ with and without further substrate}

\section{heating}

For DIP films on heated substrates $\left(100^{\circ} \mathrm{C}\right)$ covered with $\mathrm{C}_{60}$ with and without further substrate heating AFM and GISAXS measurements are performed to detect the in-plane structures. AFM again exhibits two characteristic lateral lengths, whereas in GISAXS a third lateral structure is found. For the structures seen with AFM and GISAXS, a decreasing domain size and domain distance is found with increasing substrate temperature during $\mathrm{C}_{60}$ evaporation, starting from a value similar to the domain size of the neat DIP layer evaporated at $100{ }^{\circ} \mathrm{C} . \mathrm{C}_{60}$ evaporation shows different influence on the underlying DIP layer depending on the substrate temperature. While $\mathrm{C}_{60}$ just overgrows the DIP for the unheated substrate preserving the underlying morphology (see Fig 2.c,d), it leads to reorganization of the DIP film in the case of a heated substrate during $\mathrm{C}_{60}$ evaporation (see Fig. 2.e). This cannot be observed in AFM measurements as DIP is covered by $\mathrm{C}_{60}$, but GISAXS measurements show shrinking lateral domain distances (see Fig. 5), while the vertical component is preserved (see Tab. 1). Additionally, $\mathrm{C}_{60}$ growth changes, i.e. it forms films with higher crystallinity, as probed by XRR. These measurements show enhanced domain sizes of about $46 \mathrm{~nm}$ (best crystalline coherence in $\mathrm{C}_{60}$ for the presented samples) in vertical direction compared to approximately $22 \mathrm{~nm}$ in the case of the sample without substrate heating during deposition of $\mathrm{C}_{60}$. These findings are a remarkable example, that the morphology in heterolayers cannot be predicted based on measurements performed on the underlying neat layer, even for the same 
substrates. Unexpected behavior of PV cell characteristics could thus possibly be assigned to unknown layer reorganization during deposition of top layers.

Regarding roughness correlation a different behavior as compared to the neat DIP films is seen. For large structures in the films (from vertical cuts at $q_{\mathrm{y}}=0$ ), a correlated roughness is present after $\mathrm{C}_{60}$ evaporation for room temperature and $100{ }^{\circ} \mathrm{C}$. In addition, for small particles, as obtained from the oscillations in the $q_{\mathrm{z}}$-cut at the position of the maximum in $q_{\mathrm{y}}$, a correlated roughness is obtained which is even more pronounced than for the larger structures.

\section{c) Planar-mixed architecture (thick co-evaporated mixture of DIP and $\mathrm{C}_{60}$ on DIP)}

For the planar-mixed layer system, XRR reveals a vertical coherence length of about $48 \mathrm{~nm}$ for DIP and $27 \mathrm{~nm}$ for $\mathrm{C}_{60}$. These values are comparable to the domain size in the unheated bilayer system.

AFM measurements show a different morphology as compared to all the other samples. Instead of sphere or granule-like domains, the morphology can be described as large interconnected domains with small sphere-like objects being placed on top.

FFT analysis of the AFM data reveals that the spongy shape of the underlying DIP exhibits a domain distance of about $700 \mathrm{~nm}$. Obviously small $\mathrm{C}_{60}$ grains are distributed within the DIP host structure with a mean distance of about $240 \mathrm{~nm}$. The GISAXS data analysis results in two dominant lateral lengths as well. In comparison to the planar layer system larger structures are detected with a different shape, as it is apparent from the AFM measurements, too.

In addition to the interpretation via EIA, the GISAXS data of the planar-mixed layer system has been simulated with IsGISAXS ${ }^{[27]}$ (not shown), based on the Distorted Wave Born Approximation (DWBA) in the framework of the local monodisperse approximation (LMA), resulting from monodisperse subsystems. ${ }^{[31]}$ The peak position of the interference function corresponds to a domain distance of $120 \mathrm{~nm}$ as determined with EIA. The used model can be understood as spherically shaped $\mathrm{C}_{60}$ domains with a radius of $23 \mathrm{~nm}$ mixed with cylindrically shaped DIP domains with a radius of $89 \mathrm{~nm}$ and a mean distance of $120 \mathrm{~nm}$. As the mean distance for the DIP domains is smaller than their diameter, the DIP domains are agglomerated, in agreement with the AFM data. The height of the $\mathrm{C}_{60}$ domains as obtained from the simulation is $50 \mathrm{~nm}$, while it is about $180 \mathrm{~nm}$ for the DIP. We note that the mentioned values for domain heights and radii of $\mathrm{C}_{60}$ and DIP are simulation parameters that 
have been determined by a cumulative fit of several $q_{\mathrm{y}^{-}}$and $q_{\mathrm{z}}$-cuts of the simulation results to measured data. In comparison with the vertical domain sizes as obtained from XRR as $27 \mathrm{~nm}$ and $48 \mathrm{~nm}$, the domain heights determined with the IsGISAXS simulation are larger by a factor of 2 and 4, respectively. This difference indicates that the spherically shaped $\mathrm{C}_{60}$ domains are in average present at a finite height above the substrate and that DIP is present below and between the $\mathrm{C}_{60}$ domains. Tab. 1 summarizes the comparison between values obtained by GISAXS for the sample volume and values obtained by AFM for the sample surface. For both, the large structures in the film, and for the small particles, correlated roughness is observed in case of coevaporation of DIP and $\mathrm{C}_{60}$.

The structural information gained from GISAXS, XRR and AFM measurements can now be converted to a concept how DIP and $\mathrm{C}_{60}$ grow in the respective OPVC devices (see Figure 6). For subsequent evaporation of DIP and $\mathrm{C}_{60}$ at RT, small crystallites of DIP and $\mathrm{C}_{60}$ are present on top of each other. A higher substrate temperature during evaporation of DIP results in larger DIP crystallites, while a higher substrate temperature during $\mathrm{C}_{60}$ evaporation forces the underlying DIP to reorganize and reduces its crystallite size; in contrast, $\mathrm{C}_{60}$ crystallites grow larger. In the case of coevaporation, $\mathrm{C}_{60}$ crystallites are incorporated into the spongy morphology of interconnected huge DIP crystallites. In all cases, the vertical dimensions of the DIP crystallites are as large as the film thickness.

\section{d) Solar cell performance}

To compare results from morphology and structure investigations to the solar cell performance, the growth behavior has to be similar on the two different substrates used. Comparing our results (see Fig. 2) with published data (see Ref. ${ }^{[20]}$ ) this necessary precondition is fulfilled for DIP grown on $\mathrm{SiO}_{2}$ and ITO/PEDOT:PSS. Also the values for vertical domain distances (see Tab. 1) show similar crystallization behavior on both substrates in agreement with the results from Ref. ${ }^{[20]}$. However, we note that for technical reasons related to GISAXS, the $\mathrm{C}_{60}$ layers are thinner in the present study and thus not optimized for OPVC performance.

As mentioned in the introduction, morphology and structure are critically influencing the solar cell performance. Due to the strong changes of lateral feature sizes in the layers caused by different fabrication conditions, variations in the characteristic values for the corresponding OPVCs are expected. Thus, it is even more surprising that the solar cells without heating 
during $\mathrm{C}_{60}$ deposition do not differ significantly, except for the fill factor which slightly decreases for lower substrate temperatures during deposition of DIP caused by a prospective s-shape in $j$ - $V$ characteristics, see Figure 7 and Table 2. In this case the formation of s-shaped $j-V$ characteristics results from a less pronounced increase of forward current in the first quadrant. This can mainly be attributed to an injection barrier at the PEDOT:PSS/donor interface, which is reduced by heating the substrate due to an increase of the work function of PEDOT:PSS. For that reason the substrates of the samples without heating and heating at 60 ${ }^{\circ} \mathrm{C}$ during deposition of DIP were pre-annealed to $100{ }^{\circ} \mathrm{C}$ in the vaccum chamber and cooled down to the distinct temperature before DIP film deposition, otherwise the s-shape in the $j-V$ characteristics would be more pronounced and would influence the FF of the solar cells severely. For detailed information about s-shapes in DIP/C 60 solar cells see Ref. ${ }^{[32]}$. Apart from these minor changes in fill factor by s-shapes, $j-V$ characteristics are almost identical despite the fact that lateral domain distances vary by a factor of around 3 between DIP layers prepared on unheated and heated $\left(100{ }^{\circ} \mathrm{C}\right)$ substrates. For DIP on unheated substrates lateral electron and hole mobilities are in the range of $10^{-1} \mathrm{~cm}^{2} / \mathrm{Vs}$ and $10^{-2} \mathrm{~cm}^{2} / \mathrm{Vs}^{[33]}$, respectively, and will even be better for larger domain sizes. However, being an in-plane property of the film, minor influence on OPVC performance is to be expected, as charge carrier transport and diffusion processes are dominated by film properties along the layer normal. Out-of-plane structural properties stay nearly the same, i.e. the vertical DIP domain size is approaching the layer thickness leading to comparable transport and diffusion behavior regardless of the substrate temperature during DIP growth. $\mathrm{C}_{60}$ just overgrows the DIP layer, when the substrate is not heated during deposition of $\mathrm{C}_{60}$ comprising $20 \mathrm{~nm}$ thick crystals. Thus all structural and morphological parameters are preserved for these three samples yielding comparable characteristic values in OPVCs.

Compared to the PHJ OPVCs, where the substrate is held at room temperature during $\mathrm{C}_{60}$ depostion, the sample with further substrate heating during $\mathrm{C}_{60}$ deposition exhibits a decreased FF, which is not an effect resulting from an injection barrier, as the forward current does not show an s-shape. In fact, this seems to result from a reduced parallel resistance as the current is not saturating for negative voltages. ${ }^{[34]}$ These changes in FF are obviously related to structural and morphological changes as they were observed by GISAXS, AFM and XRR. Thus, the crucial factor can most likely be associated to the reorganization of the DIP layer, where lateral grain size is shrinking as compared to the solar cell without substrate heating during $\mathrm{C}_{60}$ deposition. However, lateral grain shrinking is probably not the only reason for changes in OPVC characteristics, as there are only small changes for the OPVCs with 
unheated substrate during $\mathrm{C}_{60}$ depostition, only differing in lateral grain distances of DIP. A possibe explanation may be found in $\mathrm{C}_{60}$ diffusing into the DIP film during the reorganization process, which leads to a larger interface and encapsulated $\mathrm{C}_{60}$ islands in the DIP film. ${ }^{[35]} \mathrm{An}$ effect like this could explain changes in all three characteristic parameters of the OPVC. Encapsulation of $\mathrm{C}_{60}$ in the DIP film leads to an increased series resistance due to trapping of charges on the islands and increases the D/A interface, which leads to enhanced recombination. While trapping of charges reduces the $\mathrm{FF}$, increased recombination rates lower $V_{\text {OC. }}$ The overall effect of the reorganization process would direct the characteristic values towards those of a PM-HJ. This can be seen in the $j$ - $V$ characteristics (see Fig.7 and Tab.1). Due to the PM-HJ containing mainly mixed material, island formation for $\mathrm{C}_{60}$ is very pronounced, which severly reduces the FF. Compared to the PHJ cells, $V_{\mathrm{OC}}$ is slightly reduced because of the huge interface between DIP and $\mathrm{C}_{60}$, which leads to higher recombination losses. ${ }^{[21]}$ Despite these reductions, efficiency stays nearly the same in the range of standard deviation resulting from the gain of $j_{\mathrm{SC}}$. This can be explained by the fact, that the phase separated $\mathrm{BHJ}$ of DIP and $\mathrm{C}_{60}$ is composed of domains of both materials being in clsoe proximity to each other. Thus, the majority of excitons is dissociated within the range of EDL. ${ }^{[20,13,37]}$ The high crystallinity of both materials can explain the almost equal efficiencies of the PHJ and PM-HJ OPVCs, as EDLs are very high in crystalline materials and the gain of $j_{\mathrm{SC}}$ by mixing both materials is compensated by the exceptionally high FFs of PHJ devices. ${ }^{[36]}$

\section{Conclusion}

Structural and morphological issues play an important role in solar cell performance. In our study we showed how fabrication conditions can alter the lateral and vertical crystalline coherence in thin films of DIP and $\mathrm{C}_{60}$. In the literature, typically effects such as structural differences in materials grown on different substrates are taken into account, whereas effects which are caused by a reorganization of the underlying films are often neglected. Our structural and morphological investigations show that these reorganization processes can be very pronounced and lead to noticable changes in electronic devices, rendering them an important issue that needs to be considered. However, advanced scattering techniques such as GISAXS are required to detect such changes in buried layers.

Particularly, neat DIP layers deposited on differently heated substrates yield increasing lateral domain distances for higher temperatures. Depending on the further temperature treatment of the substrate during $\mathrm{C}_{60}$ deposition, the acceptor can simply overgrow the DIP topography 
(unheated substrate) or result in significant reorganization of the underlying DIP layer accompanied by higher crystallinity in vertical direction (heated substrate). This reorganization does not only influence the respective layer structure, but, interpreting the $j-V$ characteristics of equivalent organic solar cells, gives rise to the assumption that also intermixing of the two materials at the interface takes place. In all neat films and bilayer systems surface morphology measured by AFM and bulk structure measured by GISAXS exhibit nearly the same lateral domain distances, which is also an indication for $\mathrm{C}_{60}$ growing with correlated roughness on DIP. In contrast, domain distances extracted from surface and bulk measurements are quite different in planar-mixed layer systems indicating a completely different growth behavior. This results in DIP forming interconnected sponge-like structures sourrounded by $\mathrm{C}_{60}$ agglomerations, leading to significant changes in $j$ - $V$ characteristics most importantly a reduction of the fill factor - as compared to the bilayer systems. This is a clear signature of increased recombination losses as a consequence of an unfavourable morphology for charge carrier extraction from mixed donor-acceptor structures.

\section{Acknowledgments}

Financial support by the Deutsche Forschungsgemeinschaft within the priority program SPP 1355 'Elementary Processes of Organic Photovoltaics' (MU1487/13 and BR1728/8), the Elite Network of Bavaria within the International Graduate School 'Materials Science of Complex Interfaces' and by the Bavarian Collaborative Research Project 'Solar Technologies Go Hybrid' (SolTec) is gratefully acknowledged. Part of this research was carried out at the synchrotron light source DORIS III at DESY. DESY is a member of the Helmholtz Association (HGF). 


\section{Tables}

\begin{tabular}{|c|c||c|c|c|c|}
\hline $\begin{array}{c}\text { Layer } \\
\text { Sequence } \\
(\text { Thickness }[\mathrm{nm}])\end{array}$ & Temp. & $\begin{array}{c}\text { Lat. dom. dis. } \\
(\mathrm{GISAXS}) \\
{\left[{ }^{\circ} \mathrm{C}\right]}\end{array}$ & $\begin{array}{c}\text { Lat. dom. dis. } \\
(\mathrm{AFM}) \\
{[\mathrm{nm}]}\end{array}$ & $\begin{array}{c}\mathrm{R}_{\text {rms }} \\
(\mathrm{AFM}) \\
{[\mathrm{nm}]}\end{array}$ & $\begin{array}{c}\text { Vert. cor. len. } \\
(\mathrm{XRR}) \\
{[\mathrm{nm}]}\end{array}$ \\
\hline \hline $\mathrm{DIP}(50)$ & $\mathrm{RT}$ & 45,160 & 65,151 & 4.5 & 53 \\
\hline $\mathrm{DIP}(50)$ & 60 & 80,205 & 89,181 & 6.0 & 51 \\
\hline $\mathrm{DIP}(50)$ & 100 & 205,430 & 171,416 & 3.9 & 54 \\
\hline $\mathrm{DIP}(50) / \mathrm{C}_{60}(40)$ & $100 / \mathrm{RT}$ & $12,205,430$ & 217,436 & 6.7 & $49 / 22$ \\
\hline $\mathrm{DIP}(50) / \mathrm{C}_{60}(40)$ & $100 / 100$ & $15,110,170$ & 110,193 & 5.3 & $49 / 46$ \\
\hline $\mathrm{DIP}(4) / \mathrm{DIP}: \mathrm{C}_{60}(36)$ & $100 / 100$ & 120,550 & 243,697 & 6.2 & $48 / 27$ \\
\hline
\end{tabular}

Table 1: Structural and topographical measurements were performed on samples with DIP, DIP/C 60 and DIP/DIP: $\mathrm{C}_{60}$ layers deposited on $\mathrm{SiO}_{2}$ substrates at different temperatures as specified in the second column. EIA fits of GISAXS data and FFT fits of AFM data yield lateral domain distances with an error of $\pm 3 \%$ and $\pm 7 \%$, respectively. The same accuracy of $\pm 7 \%$ is given for $\mathrm{R}_{\mathrm{rms}}$ of AFM data, while the Gaussian fits of XRR data reveal vertical coherence lenghts with an error of about $\pm 10 \%$. The given lengths in the Table have been obtained for the particular series of samples shown in Figures 2-5. Note that the less prominent second and third length scale are not easily deconvoluted in every sample. 


\begin{tabular}{|c|c|c|c|c|c|}
\hline $\begin{array}{c}\text { Layer } \\
\begin{array}{c}\text { Sequence } \\
\text { (Thickness }[\mathrm{nm}])\end{array}\end{array}$ & $\begin{array}{c}\text { Temp. } \\
{\left[{ }^{\circ} \mathrm{C}\right]}\end{array}$ & $\mathrm{j}_{\mathrm{SC}}$ & $\begin{array}{c}\mathrm{V}_{\mathrm{OC}} \\
{[\mathrm{mA} / \mathrm{cm}]}\end{array}$ & $\begin{array}{c}\mathrm{FF} \\
{[\mathrm{V}]}\end{array}$ & $\begin{array}{c}\eta \\
{[\%]}\end{array}$ \\
\hline $\mathrm{DIP}(50) / \mathrm{C}_{60}(40)$ & $\mathrm{RT} / \mathrm{RT}$ & $3.05 \pm 0.22$ & $0.915 \pm 0.002$ & $65.6 \pm 1.0$ & $1.83 \pm 0.07$ \\
\hline $\mathrm{DIP}(50) / \mathrm{C}_{60}(40)$ & $60 / \mathrm{RT}$ & $3.04 \pm 0.12$ & $0.913 \pm 0.002$ & $66.1 \pm 0.6$ & $1.84 \pm 0.07$ \\
\hline $\mathrm{DIP}(50) / \mathrm{C}_{60}(40)$ & $100 / \mathrm{RT}$ & $3.20 \pm 0.14$ & $0.907 \pm 0.002$ & $68.3 \pm 0.4$ & $1.98 \pm 0.08$ \\
\hline $\mathrm{DIP}(50) / \mathrm{C}_{60}(40)$ & $100 / 100$ & $3.16 \pm 0.17$ & $0.902 \pm 0.008$ & $61.1 \pm 1.1$ & $1.74 \pm 0.11$ \\
\hline $\mathrm{DIP}(4) / \mathrm{DIP}: \mathrm{C}_{60}(36) / \mathrm{C}_{60}(6)$ & $100 / 100 / \mathrm{RT}$ & $4.41 \pm 0.06$ & $0.881 \pm 0.005$ & $44.1 \pm 1.2$ & $1.71 \pm 0.02$ \\
\hline
\end{tabular}

Table 2: Characteristic device parameters of organic photovoltaic cells with active layer stacks corresponding to the samples listed in Tab. 1. In this case, the organic layers were deposited on ITO/PEDOT:PSS substrates with a BCP/Al top electrode. The electrical measurements were performed under simulated AM 1.5 conditions. 


\section{References}

[1] N. S. Sariciftci, Bound neutral excitons or charged polaron pairs, in Primary photoexcitations in conjugated polymers: Molecular excitons versus semiconductor band model, World Scientific, Singapore 1997.

[2] R. R. Lunt, N. C. Giebink, A. A. Belak, J. B. Benzinger and S. R. Forrest, J. Appl. Phys. 2009, 105, 053711.

[3] S. Banerjee, A. P. Parhi, S. S. K. Iyer, S. Kumar, Appl. Phys. Lett. 2009, 94, 223303.

[4] D. Qin, P. Gu, R. S. Dhar, S. G. Razavipour and D. Ban, Phys. Stat. Sol. A 2011, 208, 1967.

[5] D. Kurrle and J. Pflaum, Appl. Phys. Lett. 2008, 92, 133306.

[6] R. W. Boer, A. F. Stassen, M. F. Craciun, C. L. Mulder, A. Molinari, S. Rogge and A. F. Morpurgo, Appl. Phys. Lett. 2005, 86, 262109.

[7] H. L. Kwok, Structure 2003, 5, 62.

[8] C. W. Tang, Appl. Phys. Lett. 1986, 48, 183.

[9] A. Foertig, A. Wagenpfahl, T. Gerbich, D. Cheyns, V. Dyakonov and C. Deibel, Adv. Energy Mater. 2012, early view.

[10] R. A. Street, M. Schoendor, A. Roy and J. H. Lee, Phys. Rev. B 2010, 81, 205307.

[11] C. W. Schlenker and M. E. Thompson, Chem. Com. 2011, 47, 3702.

[12] J. J. M. Halls, C. A. Walsh, N. C. Greenham, E. A. Marseglia, R. H. Friend, S. C. Morattia and A. B. Holmes, Nature 1995, 376, 498.

[13] G. Yu, J. Gao, J. C. Hummelen, F. Wudl, A. J. Heeger, Science 1995, 270, 1789.

[14] T. Tsuzuki, Y. Shirota, J. Rostalski and D. Meissner, Sol. Energy Mat. Solar C. 2000, 61,1 .

[15] A. Opitz, J. Wagner, W. Brütting, I. Salzmann, N. Koch, J. Manara, J. Pflaum, A. Hinderhofer and F. Schreiber, IEEE J. Sel. Top. Quant. 2010, 16, 1707.

[16] A. Hinderhofer and F. Schreiber, Chem. Phys. Chem. 2012, 13, 628.

[17] A. C. Dürr, F. Schreiber, M. Münch, N. Karl, B. Krause, V. Kruppa and H. Dosch, Appl. Phys. Lett. 2002, 81, 2276.

[18] A. C. Dürr, F. Schreiber, K. A. Ritley, V. Kruppa, J. Krug, H. Dosch and B. Struth, Phys. Rev. Lett. 2003, 90, 016104. 
[19] S. Kowarik, A. Gerlach, S. Sellner, F. Schreiber, L. Cavalcanti and O. Konovalov, Phys. Rev. Lett. 2006, 96, 125504.

[20] J. Wagner, M. Gruber, A. Hinderhofer, A. Wilke, B. Bröker, J. Frisch, P. Amsalem, A. Vollmer, A. Opitz, N. Koch, F. Schreiber and W. Brütting, Adv. Funct. Mater. 2010, 20, 4295.

[21] M. Gruber, J. Wagner, K. Klein, U. Hörmann, A. Opitz, M. Stutzmann and W. Brütting, Adv. Energy Mater. 2012, 2, 1100.

[22] P. Müller-Buschbaum, Anal. Bioanal.Chem. 2003, 376, 3.

[23] J. Huang, J. Yu, H. Lin and Y. Jiang, J. Appl. Phys. 2009, 105, 073105.

[24] T. Osasa, S. Yamamoto and M. Matsumura, Jpn. J. Appl. Phys. 2006, 45, 3762.

[25] G. Renaud, R. Lazarri and F. Leroy, Surf. Sci. Rep. 2009, 64, 255.

[26] S. V. Roth, R. Döhrmann, M. Dommach, M. Kuhlmann, I. Kröger, R. Gehrke, H. Walter, C. Schroer, B. Lengeler and P. Müller-Buschbaum, Rev. Sci. Instrum. 2006, 77, 085106.

[27] R. Lazzari, J. Appl. Cryst. 2002, 35, 406.

[28] A. Hinderhofer, T. Hosokai, K. Yonezawa, A. Gerlach, K. Kato, K. Broch, C. Frank, J. Novak, S. Kera, N. Ueno and F. Schreiber, Appl. Phys. Lett. 2012, 101, 033307.

[29] P. Müller-Buschbaum and M. Stamm, Macromolecules 1998, 31, 3686.

[30] P. Müller-Buschbaum, J. S. Gutmann, C. Lorenz, T. Schmitt and M. Stamm, Macromolecules 1998, 31, 9265.

[31] G. Kaune, M. A. Ruderer, E. Metwalli, W. Wang, S. Couet, K. Schlage, R. Röhlsberger, S. V. Roth and P. Müller-Buschbaum, ACS Appl. Mater. Interfaces 2009, 1, 353. [32] J. Wagner, M. Gruber, A. Wilke, Y. Tanaka, K. Topczak, A. Steindamm, U. Hörmann, A. Opitz, Y. Nakayama, H. Ishii, J. Pflaum, N. Koch and W. Brütting, J. Appl. Phys. 2012, $111,054509$.

[33] M. Horlet, M. Kraus, W. Brütting and A. Opitz, Appl. Phys. Lett. 2011, 98, 233304.

[34] P. Würfel, Physics of Solar Cells, Wiley-VCH, Weinheim, Germany 2005.

[35] P. Peumans, S. Uchida and S. R. Forrest, Nature 2003, 425, 158.

[36] B. Ray, M. S. Lundstrom and M. A. Alam, Appl. Phys. Lett. 2012, 100, 013307.

[37] N. S. Sariciftci, L. Smilowitz, A. J. Heeger and F. Wudl, Science 1992, 258, 1474. 


\section{Figures}
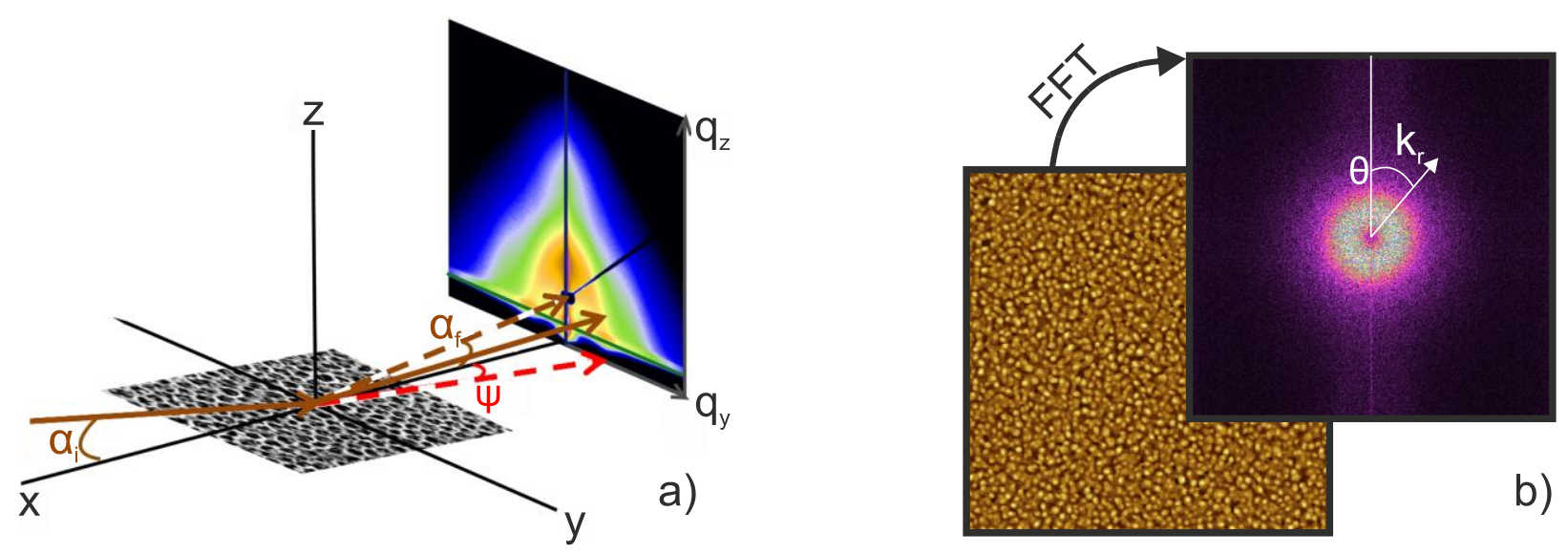

Figure 1: a) Sketch of the GISAXS setup, the beam impinges on the sample under a very shallow angle $\alpha_{i}$ and exits the sample under an angle $\alpha_{\mathrm{f}}$ in the scattering plane (xz-plane) and an angle $\Psi$ out of the scattering plane (xy-plane). b) Sketch of the fast Fourier transformation (FFT) analysis of the AFM images. 
a)

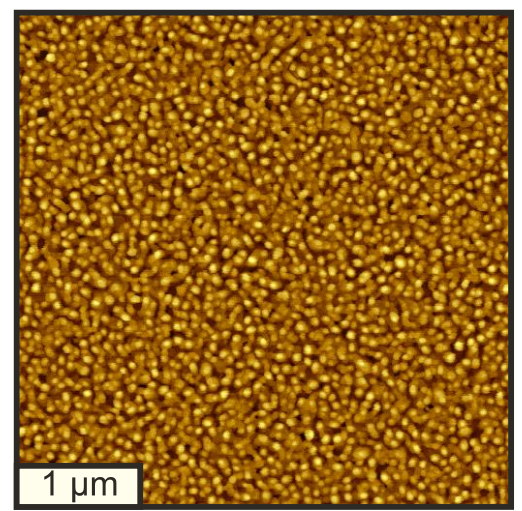

$\operatorname{DIP}(\mathrm{RT})$

b)

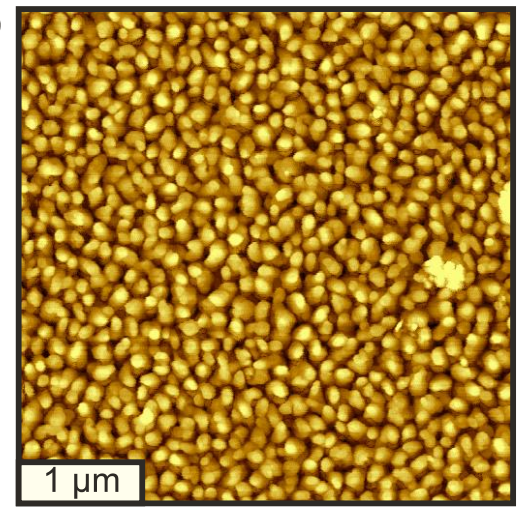

$\operatorname{DIP}\left(60^{\circ} \mathrm{C}\right)$

c)

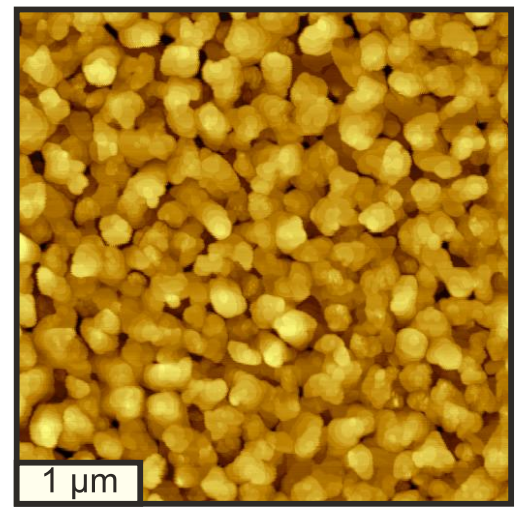

$\operatorname{DIP}\left(100^{\circ} \mathrm{C}\right)$
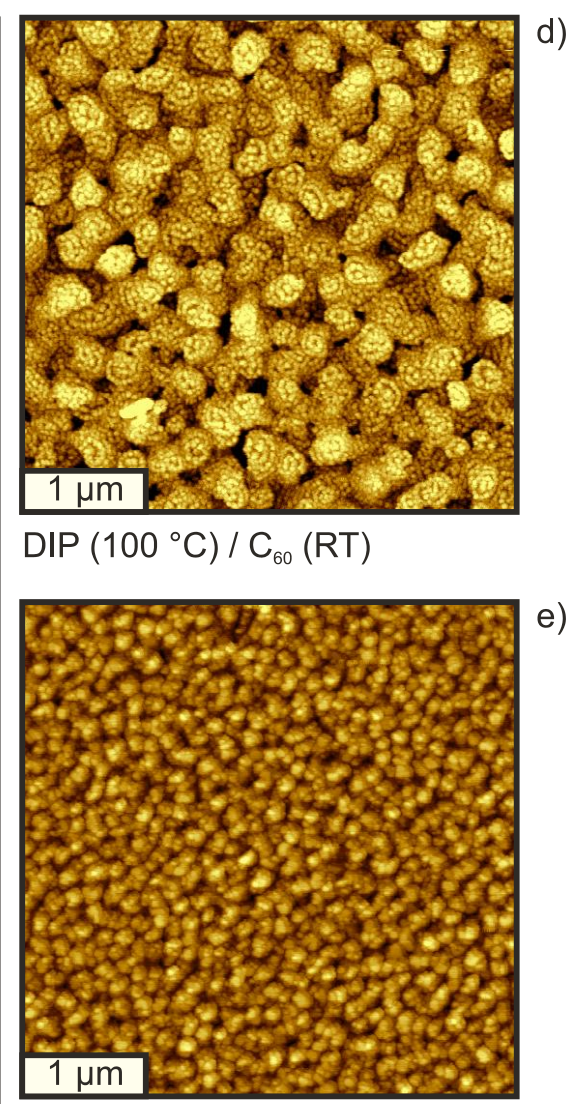

$\operatorname{DIP}\left(100^{\circ} \mathrm{C}\right) / \mathrm{C}_{60}\left(100^{\circ} \mathrm{C}\right)$

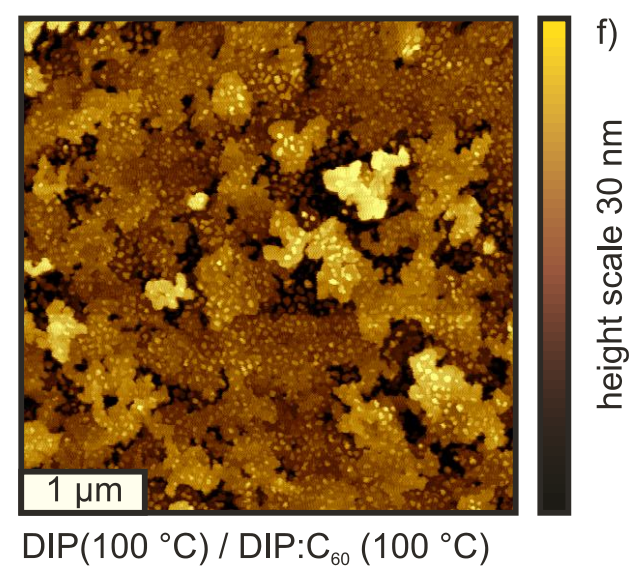

Figure 2: AFM topography images of neat DIP films on unheated and heated substrates (a-c), bilayers with $\mathrm{C}_{60}$ on top of DIP for unheated and heated substrates during $\mathrm{C}_{60}$ deposition $(\mathrm{d}, \mathrm{e})$ and a 1:1 mixed layer of DIP and DIP: $\mathrm{C}_{60}$ on top of a thin DIP seed layer on a heated substrate (f). 


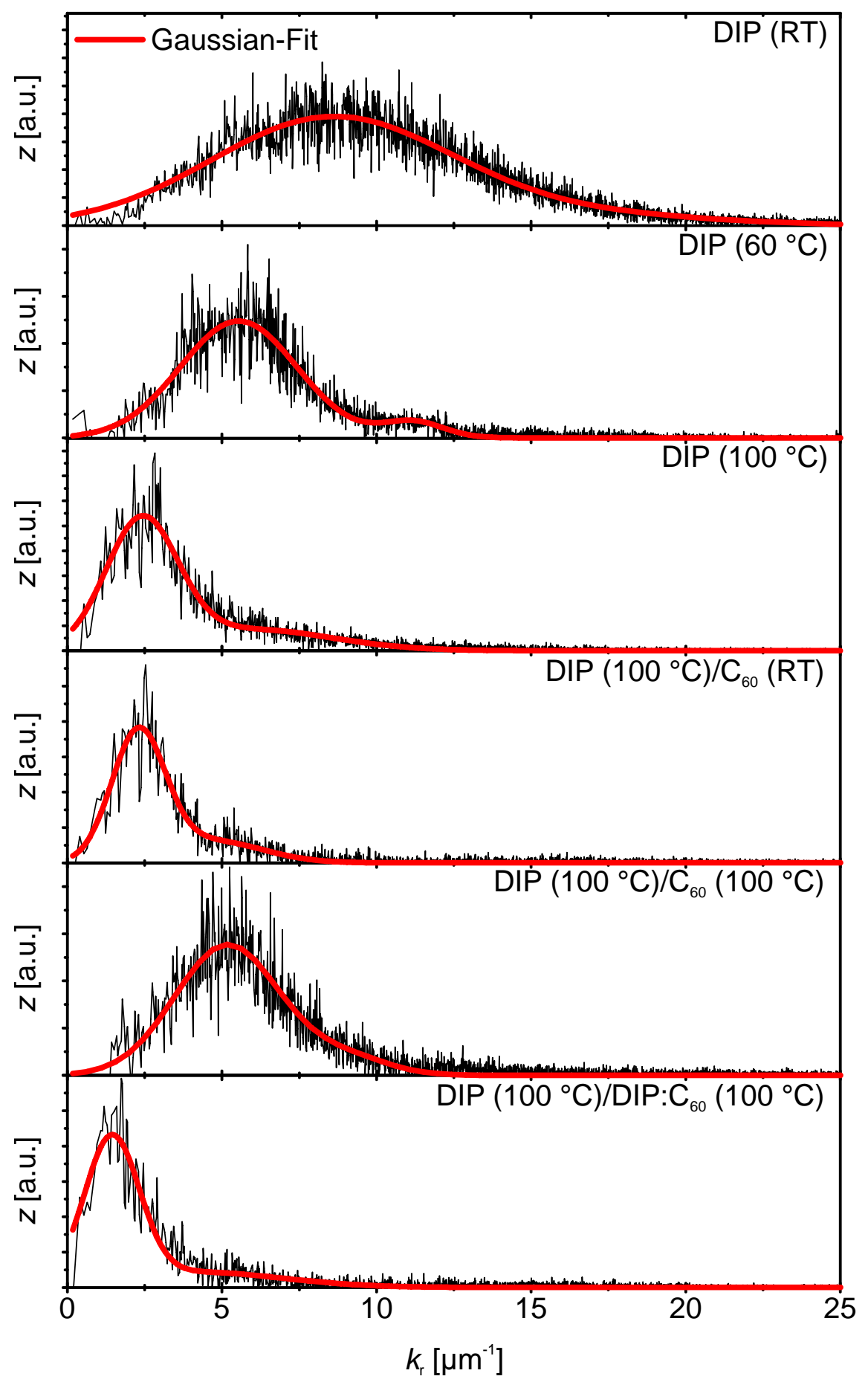

Figure 3: Angle averaged FFT of the corresponding AFM images shown in Fig. 2 fitted with two Gaussian distributions each leading to average domain distances summarized in Tab. 1. 


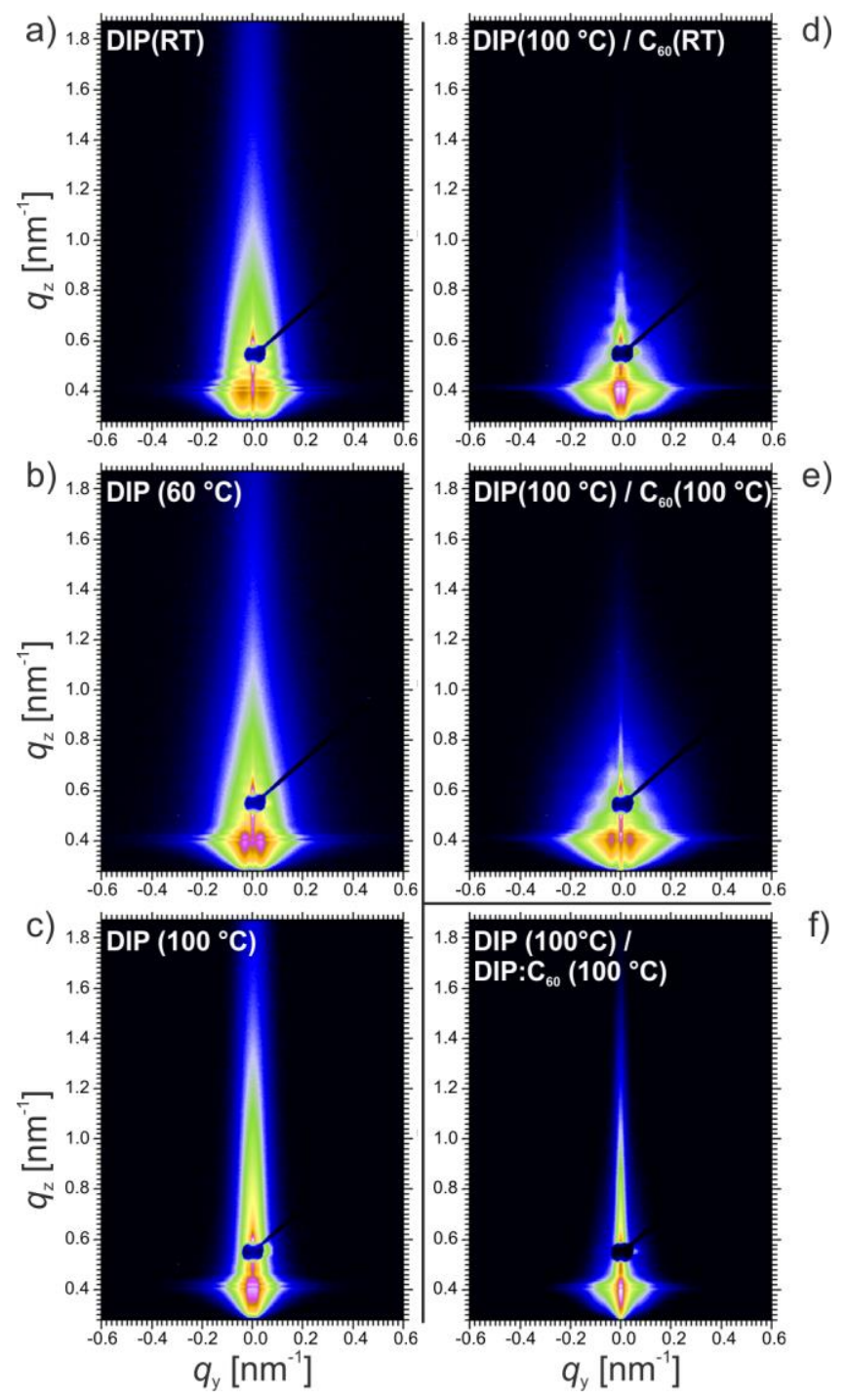

Figure 4: 2d-GISAXS data of neat DIP films on heated substrates (a-c), bilayers with $\mathrm{C}_{60}$ on top of DIP for unheated and heated substrates during $\mathrm{C}_{60}$ deposition $(\mathrm{d}, \mathrm{e})$ and a 1:1 mixed layer of DIP and $\mathrm{C}_{60}$ on top of a thin DIP seed layer on a heated substrate (f). 


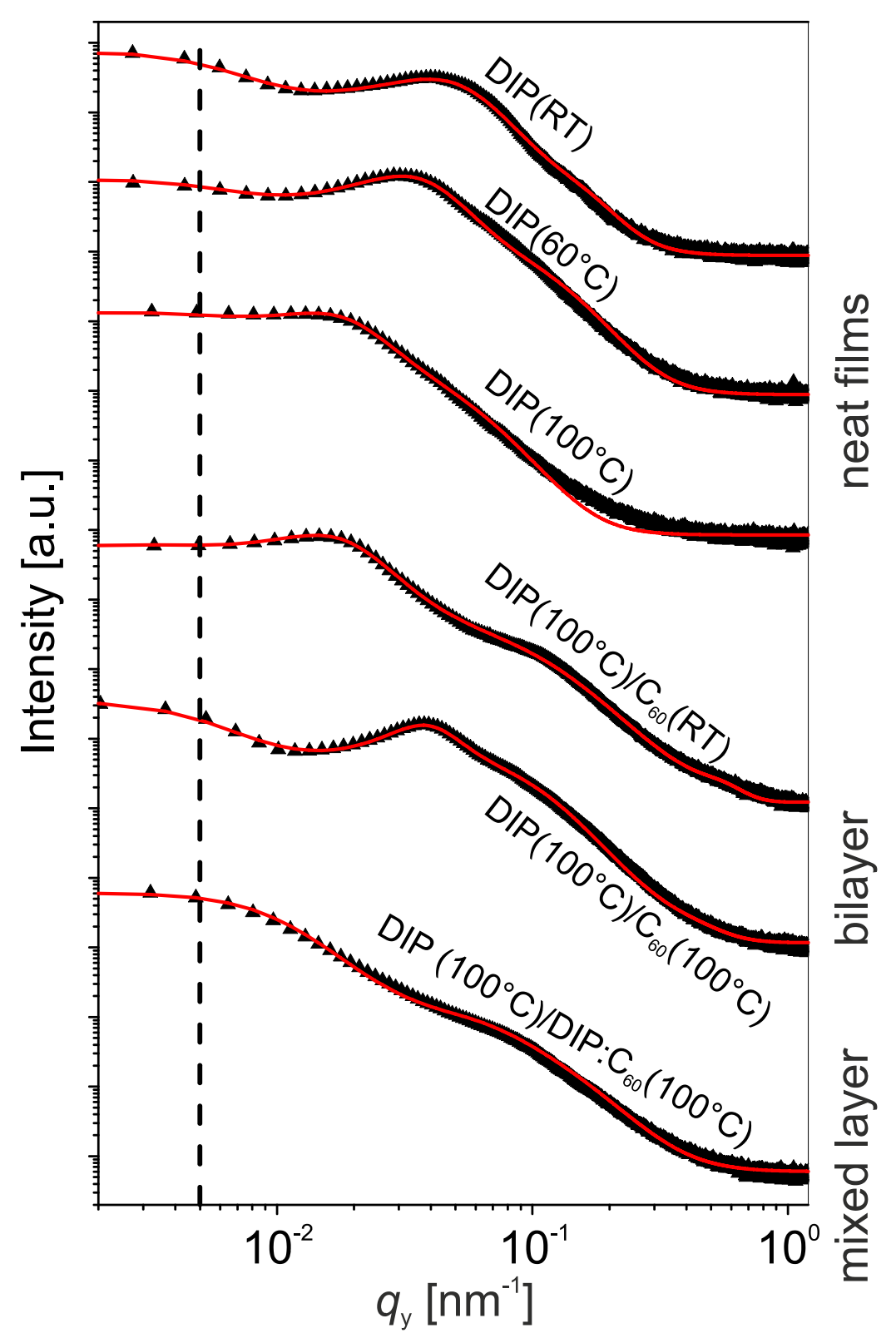

Figure 5: Horizontal line cuts of the 2d-GISAXS data at the critical angle fitted with EIA providing lateral domain distances in the bulk of the films. The curves for the neat DIP film grown at RT, the two bilayers and the mixed layer are fitted with two values for lateral domain distances, while the remaining two curves for heated DIP neat films can only be fitted accurately with three values. Curves are shifted along the intensity axis for clarity. 

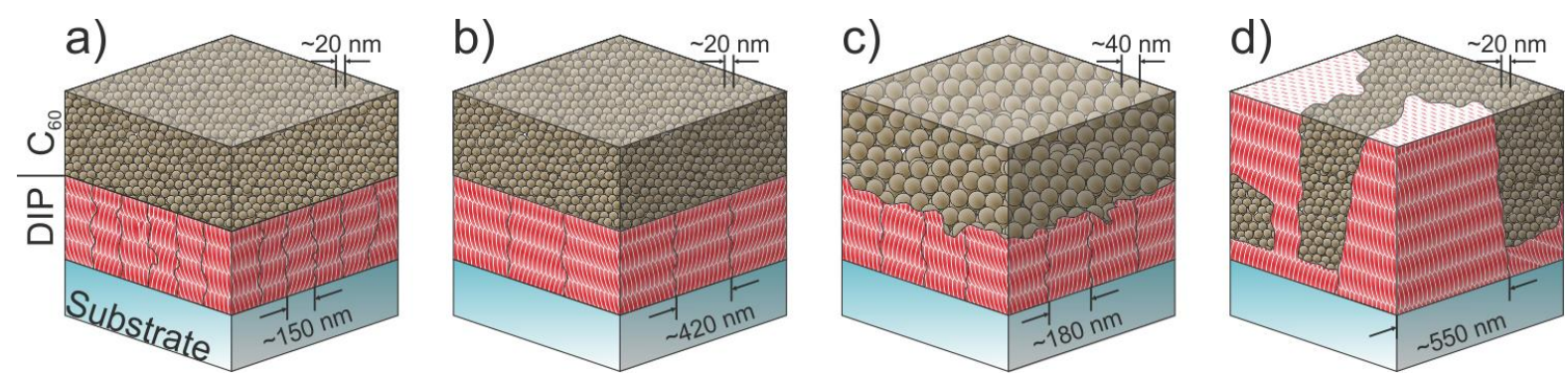

Figure 6: Concept of crystallization in $\mathrm{DIP} / \mathrm{C}_{60}$ organic solar cells for different types of cell architecture obtained from GISAXS, XRR and AFM measurements. a) corresponds to the sample without substrate heating during the whole deposition process, b) to the sample with substrate heating $\left(100^{\circ} \mathrm{C}\right)$ during deposition of DIP, c) to the sample with substrate heating $\left(100{ }^{\circ} \mathrm{C}\right)$ during deposition of both DIP and $\mathrm{C}_{60}$ and d) to the sample with a coevaporated bulk heterojunction of DIP: $\mathrm{C}_{60}$ on a thin DIP seed layer with substrate heating at $100{ }^{\circ} \mathrm{C}$. 


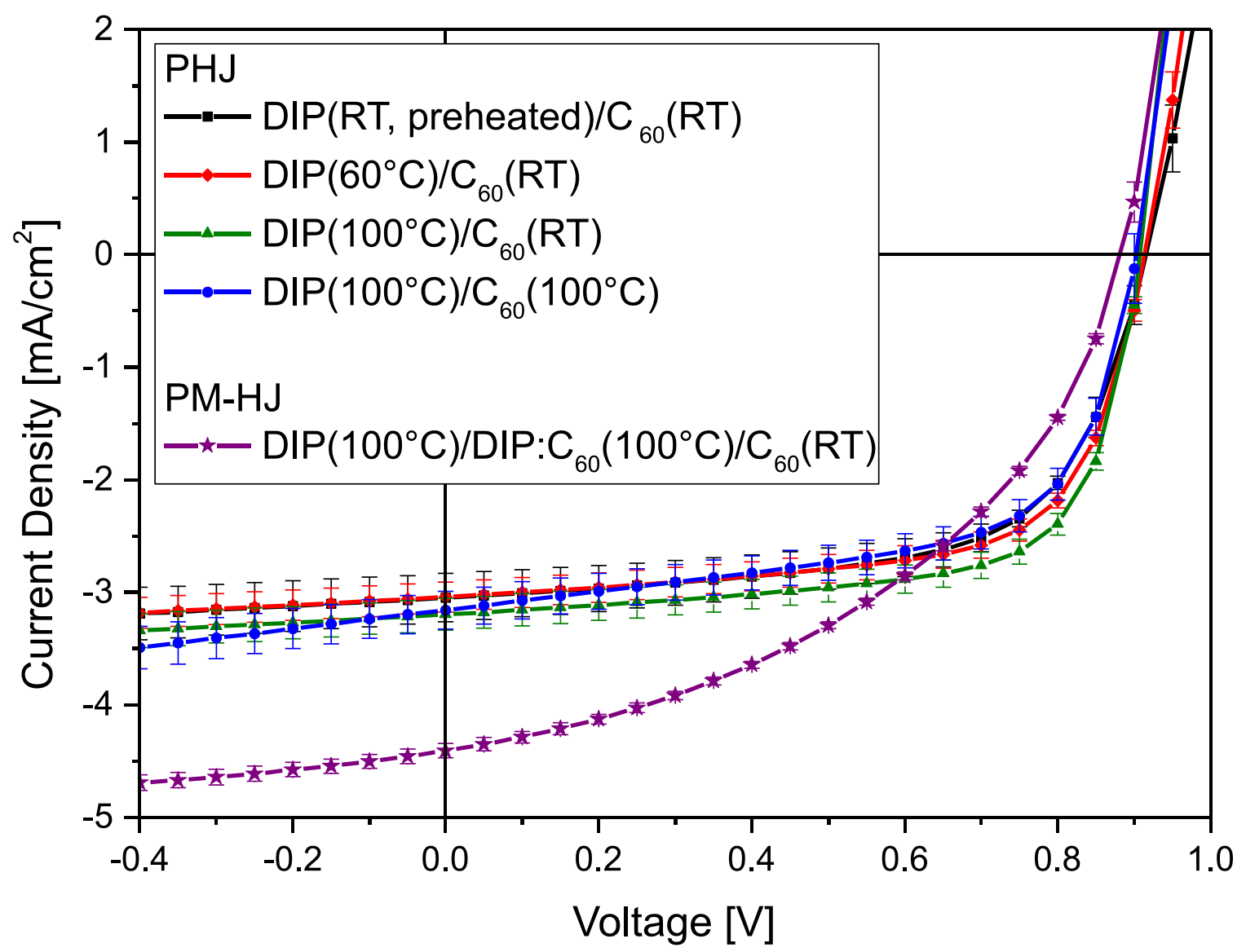

Figure 7: j-V-characteristics of organic solar cells with layer stacks comparable to the samples for structural and morphological investigations. The extracted device parameters are listed in Table 2. 
The performance of small molecule organic photovoltaic cells shows a critical dependence on morphology and structure of the active layers. It is shown how fabrication parameters, like substrate temperature and device architecture, play a significant role for crystallization and roughening of the film and how these features are related to characteristic parameters of planar and planar-mixed heterojunction donor/acceptor cells under operation.

diindenoperylene, organic solar cells, GISAXS, thin film morphology

M. Gruber, M. Rawolle, J. Wagner, D. Magerl, U. Hörmann, J. Perlich, S. V. Roth, A. Opitz, F. Schreiber, P. Müller-Buschbaum, and W. Brütting

Correlating structure and morphology to device performance of molecular organic donoracceptor photovoltaic cells based on Diindenoperylene (DIP) and $\mathrm{C}_{60}$

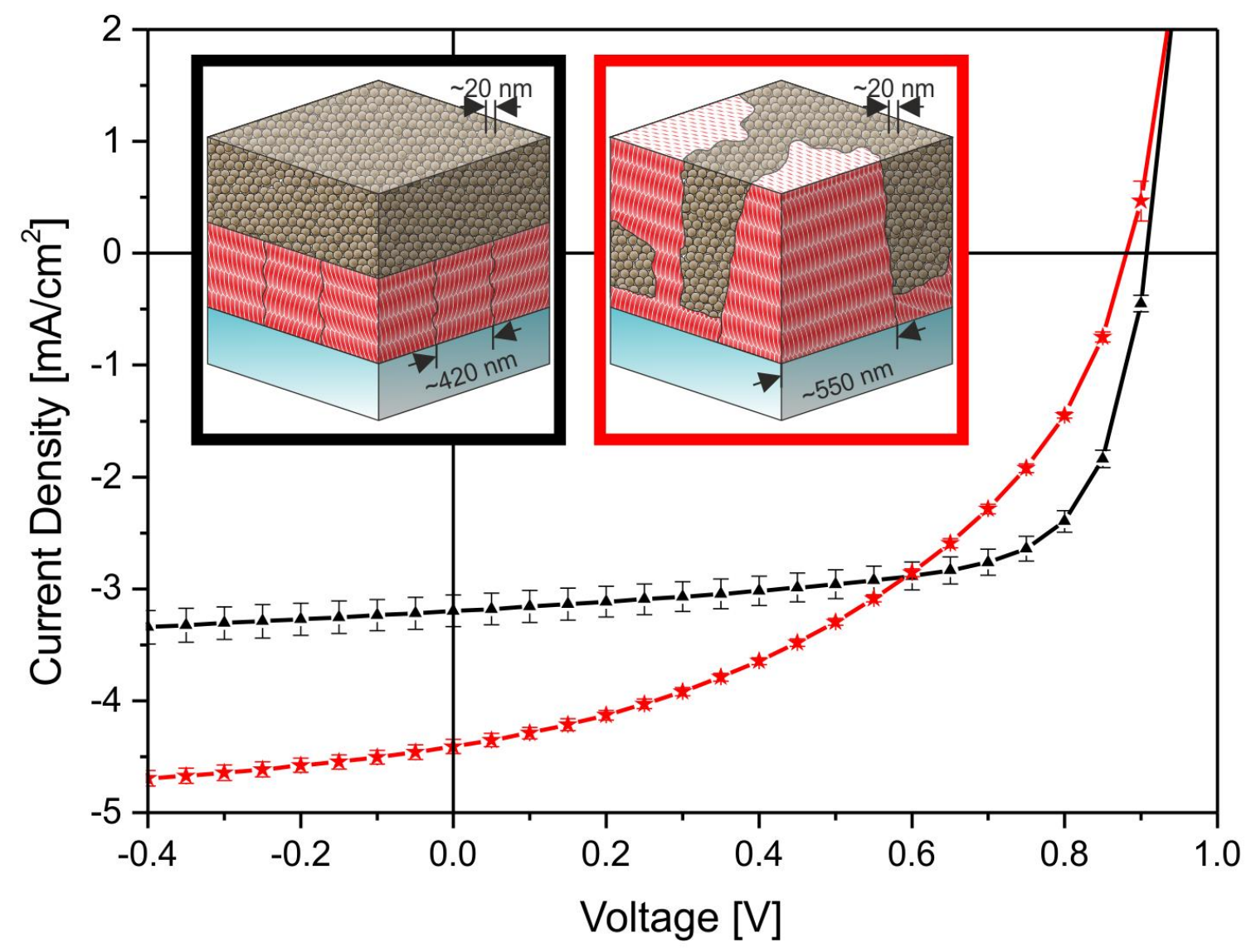

